Open Science 2.0: Large-Scale Collaborative Education Research

\author{
Matthew C. Makel, PhD \\ Duke University Talent Identification Program \\ mmakel@tip.duke.edu \\ https://orcid.org/0000-0002-3837-0088 \\ Kendal N. Smith, JD \\ Department of Educational Psychology, University of North Texas \\ Kendal.smith@unt.edu \\ https://orcid.org/0000-0002-2266-7901 \\ Matthew McBee, $\mathrm{PhD}$ \\ Department of Psychology, East Tennessee State University \\ mcbeem@etsu.edu \\ https://orcid.org/0000-0001-9122-1658 \\ Scott J. Peters, PhD \\ Department of Educational Foundations, University of Wisconsin - Whitewater \\ peterss@uww.edu \\ https://orcid.org/0000-0003-2459-3384 \\ Erin Miller, $\mathrm{PhD}$ \\ Department of Psychology, Bridgewater College \\ emmiller@bridewater.edu \\ https://orcid.org/0000-0002-9448-037X
}




\begin{abstract}
Concerns about the replication crisis and false findings have spread through a number of fields, including educational and psychological research. In some pockets, education has begun to adopt open science reforms that have proven useful in other fields. These include preregistration, open materials and data, and registered reports. These reforms are necessary and offer education research a path to increased credibility and social impact. But they all operate at the level of individual researchers' behavior. In this paper, we discuss models of large-scale collaborative research practices and how they can be applied to educational research. The combination of large-scale collaboration with open and transparent research practices offers education researchers an exciting new method for falsifying theories, verifying what we know, resolving disagreements, and exploring new questions.
\end{abstract}

Keywords: Credibility Revolution; Open Science; Replicability Crisis 


\section{Open Science 2.0: Large-Scale Collaborative Education Research}

The gulf between what research evidence suggests as effective and what happens in schools is a perennial concern. Bridging this gap is neither easy nor straightforward. Previous reports often focus on the slow process of "knowledge creep" or changing practitioner beliefs (NRC, 2002) and the National Academies of Sciences, Engineering, and Medicine have commissioned a joint research agenda on communicating science effectively (NRC, 2017). One explanation for the gap between research evidence and educational practice that is worth consideration is that practitioners may not perceive research findings as accurate, and thus neither trustworthy nor useful. Recent research in other fields, such as psychology (Open Science Collaboration, 2015), has revealed that many previously published findings cannot be replicated. The existence of this "replication crisis" (Pashler \& Harris, 2012) suggests that such concerns may be warranted. Consider recent findings regarding the status of celebrated ideas such as learning styles (Pashler, McDaniel, Rohrer, \& Bjork, 2009), growth mindset (Sisk, Burgoyne, Sun, Butler, \& Macnamara, 2018), grit (Crede, Tynan, \& Harms, 2017), or multiple intelligences (Waterhouse, 2006). All of these are staples of teacher training and instructional pedagogy, but their research basis is uncertain at best and "debunked" at worst.

In education, useful new knowledge is often hard to come by. Most of the studies curated by the What Works Clearinghouse produced effect size estimates in the null to tiny range. Evidence suggests that the majority of education randomized control trial results (the "gold standard" of research) do not have sufficient precision to assess whether the intervention was effective (Lortie-Forgues \& Ingles, 2019). Worse yet, replication has been a low priority. The vast majority of educational claims have never been subjected to independent direct replication attempts. When replications have happened, only $54 \%$ of independently conducted replications 
reported success at replicating the original finding (Makel \& Plucker, 2014). Thus, we in the field of education do not even know what we do not know. Like other fields, the education research literature undoubtedly contains false positives, but we know neither the rate nor the identity of these false claims.

These results are concerning when a fundamental goal of science is to help transcend our human flaws and biases to, as Carl Sagan suggested, winnow deep truths from deep nonsense (1997). More recently, Vazire (2018) noted that "[t]he common goal among all scientists is to accumulate knowledge. Much of the heat surrounding discussions about credibility can be attributed to differences in opinion about the best way to maximize this progress" (p. 415) and framed ongoing developments in methodological practice as a "credibility revolution" that could help increase scientific progress. We believe that several advances developed in other scientific fields to increase credibility serve as a wonderful opportunity for the education research community. In this paper, we briefly review evidence for the flaws of existing scientific practices, describe the open science practices that change the norms of research practice, and then introduce some existing models for large scale collaborative research. We believe that these collaborative models, when combined with open science values and practices, can help usher in a credibility revolution in education research that could help close the research-practice gap.

\section{Existing Problems in Research Practices}

The replication crisis in the social sciences has received extensive coverage in both the academic and popular press. The crisis is not rooted in new actions, but in broader awareness of longstanding weaknesses. The replication crisis began when it was discovered that many published research findings are not replicable, findings that are broadly consistent with Ioannidis' decade-earlier contention that "most research findings are false" (2005). A majority of 
physical and life scientists reported believing that there was a "significant crisis" with $90 \%$ believing that at least a "slight crisis" was occurring, and only $3 \%$ saying there was no crisis (Baker, 2016). Again, this concern appears to be grounded in reality as over $60 \%$ of respondents reported trying and failing to replicate the results of other researchers. We believe that if a scientific result cannot be replicated, it cannot be considered "true" or useful beyond the context in which it was found; one cannot construct a science of flukes or idiosyncratic one-offs. Lack of replicability of results is a crisis because a fundamental tenet of empirical science is replication; a finding can only represent something scientifically or even practically useful if it can be replicated by independent researchers.

Common research practices contributed to the replication crisis. There is a long history of publication bias favoring statistically significant results (Sterling, 1959). However, biases extend deeper than simply what studies get published to include which results are included within publications as well as which findings are cited in subsequent publications (de Vries et al., 2018). In education, Pigott and colleagues (2013) examined dissertations and their subsequent publications. Less than a quarter of the published versions contained all the outcomes reported in the dissertation. The results that were omitted did not appear at random; nonsignificant dissertation results were 1.30 times less likely to be reported in the published version than statistically significant results. This selective reporting of findings, in the form of removing the null results from published literature, creates a false impression that researchers and interventions are more consistent and successful than they actually are.

Another cause for the lack of reproducibility is the flexibility researchers have when making methodological decisions. Sometimes called researcher degrees of freedom (Simmons, Nelson, \& Simonsohn, 2011) or a garden of forking paths (Gelman \& Loken, 2013), this 
flexibility allows researchers to, wittingly or unwittingly, torture their data so that it confesses whatever may be desired. Sometimes this flexibility includes behaviors that have been labeled as questionable research practices (QRPs) such as selectively reporting outcomes, peeking at results to decide whether to collect additional data, determining data exclusion based on its impact on results, rounding p-values down so that they are below relevant thresholds, and reporting unexpected results as though they were predicted (John, Loewenstein, \& Prelec, 2012). Surveys of active researchers in several fields show that large percentages admit to engaging in QRPs (e.g., Fiedler \& Schwarz, 2016; Fraser, Parker, Nakagawa, Barnett, \& Fidler, 2018; John et al., 2012).

Researcher degrees of freedom also include the more subtle and prosaic flexibility characteristic of most research endeavors where there is almost always more than one reasonable way to measure key variables (Flake \& Fried, 2019), prepare the data (Steegen, Tuerlinckx, Gelman, \& Vanpaemel, 2016), and analyze it (Silberzahn et al., 2018). This flexibility, coupled with the quasi-exploratory nature of most educational data analysis, produces an opportunity for researchers to see many different possible focal results during the course of an analysis. If researcher behavior is altered by observing those results, the reported result could be selected for their desirability, whether consciously or unconsciously. When this occurs, the study's evidence is overstated, error-control properties of statistical inference are damaged, and the risk of falsepositive inference becomes much higher than was intended or reported.

The basic organizational unit for conducting education research has traditionally been a research group consisting of a few individuals. The result of this model is a large number of small studies, resulting in what might be described as broad but shallow information. Under this model, the field's research resources are divided across a large number of projects. Therefore, 
only a relatively small amount of resources, time, and labor can be devoted to each one. In turn, relatively little information can be gained from each study. A typical educational study, even if methodologically rigorous, conducted under this model produces weak evidence (Lortie-Forgues \& Ingles, 2019). There are exceptions, of course, but this description is true to a first approximation.

To accommodate this shallow-but-broad model, the research community's implicit standards for rigor must be relatively weak. Strong, definitive evidence is out of reach in most cases given the constraints imposed by limited resources. The acceptable standards for sample size, sampling procedures, measurement, and design are not those that can produce trustworthy and generalizable results, but instead what is feasible for small research teams to accomplish. These standards were adopted to the type of products that the organizational model could provide, not the other way around. Therefore, a typical study produced under this model might be described as yielding only circumstantial evidence or perhaps heavily-caveated suggestions for practitioners and researchers. This model also results in an evaluative framework under which the sheer quantity of publications is treated as a close proxy of researcher productivity.

The typical study performed under the "many small research teams" model is often based on a small convenience sample (of schools or classrooms, if not of students), uses relatively poor measurement, employs a weak design, and therefore nearly always suffers from unresolved threats to validity that renders the results ambiguous. For example, socioeconomic status is a central concept in educational research, but researchers have been content to accept free- or reduced-price lunch status proxy as an acceptable substitute because it is easy to collect despite its well-established questionable validity as a measure of socioeconomic status or even poverty (e.g., Greenberg, 2018; Harwell \& LeBeau, 2010). The philosophy seems to be that, though we 
are losing money on every sale, we can make it up in volume (Miller, 1988). Small sample sizes and reliance on cross-sectional, between-subjects designs means that most studies performed under this model are seriously underpowered to detect anything but strong effects (Chase \& Chase, 1976; Sedlmeier \& Gigerenzer, 1989; see also Schochet, 2008). Further, without random sampling from a defined population, the convergence properties underlying statistical estimation and inference may not apply (Hahn \& Meeker, 1991). This is a troublesome issue to be sure, but one that has been largely ignored because probability samples are beyond the reach of a small research team's resources.

The problems of underpowered studies have been under discussion for quite some time (c.f., Cohen, 1988), but absent anything beyond theoretical evidence of harm, these critiques were not persuasive enough to jolt the organization of educational science out of its comfortable equilibrium. Widespread replication failures in psychology, biomedical sciences, and other fields have reasserted the problems inherent to small, underpowered studies (Lindsay, 2015). Low statistical power and poor estimation precision create an environment in which $p$-hacking techniques and other QRPs are especially effective and indeed sometimes necessary to produce positive claims (Francis, Tanzman, \& Matthews, 2014; Schimmack, 2012). Further, these techniques interact with publication bias, resulting in substantially inflated effect size estimates (c.f., Type-M errors, see Gelman \& Carlin, 2014). Preregistered replication studies, in which most QRPs cannot be employed, have revealed that true effect sizes tend to be roughly half as large as meta-analyses of published studies would suggest (Anderson \& Maxwell, 2017; Open Science Collaboration, 2015). This means that typical social science effect sizes are likely substantially smaller than was reported. This, in turn, diminishes the incremental value of small studies even more, as they are even more underpowered than was thought. No matter how many 
bricks one might have, one cannot build a robust structure if few of the bricks can support any weight. This is why many believe that meta-analyzing the existing literature is not a credible strategy for producing trustworthy evidence (e.g., Van Elk, Matzke, Gronau, Guan, Vandekerckhove, \& Wagenmakers, 2015). Rather, reforms must implemented that will increase the value and quality of individual studies.

With so many well-grounded concerns about the veracity and generalizability of research findings within the research community, it should come as little surprise if and when such concerns extend to non-researchers. The field of educational research must strive to be worthy of public trust before it can expect it.

\section{Previously Proposed Solutions}

In 2018, three independent papers called for increased use of open science research practices in education (van der Zee \& Reich, 2018), special education (Cook, Lloyd, Mellor, Nosek, \& Therrien, 2018), and gifted education (McBee, Makel, Peters, \& Matthews, 2018) to increase the veracity and internal validity of published research. All three papers suggested that using practices such as preregistering hypotheses, sharing data and research materials openly, and making research papers freely available as often as possible would improve the quality of research in education. A fourth paper (Gehlbach \& Robinson, 2018) focused specifically on how preregistration could mitigate illusory findings in education research. These calls focus on important shifts in when various stages of work are done and how information is shared. However, these previously proposed solutions focus on behavioral changes of individual researchers. Although these all have the potential to better align practices with espoused values, some of the most effective reforms operate at higher levels of social organization such as the new NSF/IES companion guidelines on replication and reproducibility (2018) or the recently created 
registry of efficacy and effectiveness studies in education (Anderson, Spybrook, \& Maynard, 2019).

\section{Collaborative Research}

An additional approach to many of the aforementioned challenges is to pool resources to conduct a smaller number of more rigorously designed better studies through large-scale collaboration. This model has been tremendously successful in the physical and life sciences. Two recent examples of large collaborations in physics are the discoveries of gravitational waves by the LIGO and Virgo collaborations (Abbot et al., 2017; a paper featuring 1,011 authors) and the Higgs boson announced in separate papers by the ATLAS and CMS collaboration groups with over 3,000 authors each (Aad et al., 2012; Chatrchyan et al., 2012). Such megacollaborations have also been used outside of physics. The 2001 paper presenting the initial sequencing of the human genome was authored by the International Human Genome Sequencing Consortium, consisted of about 2,900 individual authors. Many individuals work on few projects, but produce rigorous, highly-informational findings. In recent years, psychologists have begun to experiment with large, ambitious, and rigorous collaborative research activities. These efforts have garnered a great deal of attention both within the academy and in the popular press (e.g., Aschwanden, 2015; Yong, 2018), and have had a major impact on the state of multiple subdisciplines of psychology, most notably social psychology. We believe that education research is well suited for such collaborations because of the large number of researchers and schools spread across the country and world, the difficulty of accessing schools and students for research participation, and the relatively small amount of funding available. This creates a context in which pooling resources is especially valuable. 
In this paper, we propose that education would benefit from greater use of large-scale research collaboration. Collaborative initiatives would improve understanding of what results are generalizable, when they are generalizable, and how they are generalizable. Perhaps more importantly, many of these initiatives will help assist schools and investigators who don't have the resources for independent large-scale evaluation. In the following sections we introduce different types of collaborative research initiatives, why they are helpful generally, review what they could do to help improve education research, and provide resources for how to begin implementing these practices (Table 1).

\section{Participating Labs Run Different Studies}

In this model, the collaboration runs a set of separate projects, with individual research studies assigned to the smaller constituent labs or research groups. The Open Science Collaboration (OSC) is an example of this type of collaborative effort. In 2015, the OSC published the results of the Reproducibility Project: Psychology (RP:P), in which 100 psychology studies from a variety of sub-disciplines were replicated using preregistered, highpowered protocols (OSC, 2015). A more recent example of this type of collaboration was used to investigate the reproducibility of social science experiments published in the journals Science and Nature from 2010-2015, and also the extent to which a betting market could classify the studies as replicable or non-replicable (Camerer et al., 2018).

This model has been typically used to answer meta-science research questions and is well-suited for replication efforts. This is because the research produced by this type of collaboration does not benefit from a large-scale concentration of resources at the individual study level. Thus, they are less well-suited to the definitive answering of new questions than to the assessment of the replicability of previous findings. The value of the findings produced by 
this model directly relate to the credibility of the individual studies. When these are preregistered, openly share data and materials, and are executed independently from the original research team, each study can be relatively trustworthy, particularly when they are direct replications of past research. In direct replications, the original design, treatment/manipulation, and measurement practices are treated as a fixed feature of the protocol rather than subject to change or optimization. The goal of this form of collaborative effort is to make inferences regarding the set of studies or findings. Thus, the study selection process plays a central role into determining what conclusions can be drawn from the pattern of findings across the studies in this model.

\section{Multi-Lab Collaboration Projects}

Multi-lab collaborations take the principle of independent replication and apply it to a single investigation - one conducted by several, independent research teams that have all agreed to follow a common protocol. The premise being that multiple teams simultaneously conducting the same study will provide a more definitive answer to whether the finding exists and how variable it might be across contexts (i.e., whether it is generalizable). Multi-lab collaborations must agree on a shared protocol (i.e., participants, methods, analyses) prior to data collection. This serves as a preregistration and thus limits researcher degrees of freedom. After the protocol is implemented, the findings from each team are reported individually as well as in aggregate to show the variability in effect.

Multi-lab collaborations do not have to be replications; they can test new questions or interventions. That said, testing previously established findings that are believed to have value can help focus what questions merit such attention and resources. One multi-lab collaboration tested 12 classic psychological findings (13 effects) to see if previously published findings could 
be replicated as well as assess the variability of the effect across samples (Klein et al., 2014). Each team followed the same specified protocol, collected data from at least 80 participants, recorded a video of their administration procedures, and documented any deviation from the established protocol. In the end there were 36 samples with 6,344 total participants. Not every research team conducted each of the 12 studies, but every study included multiple teams, thereby evaluating intra-team variance in the effects.

Multi-lab collaborations are helpful because they report the variability in effects across sites and researchers. For example, in the Klein et al. (2014) paper, the authors tested 13 effects and found support for ten. One particular effect, Currency Priming (exposing people to money makes them more likely to endorse a system or policy), did not replicate and showed a consistent effect size clustered around zero (the original study effect was closer to $d=1.0$ ). Compare this to Anchoring (estimating size or distance after first being presented by implausible values) which replicated, but showed a far wider range of effect $(d$ ranged from $<1.0$ to $>3.0)$ than previously reported.

Observed effects are influenced by a range of factors, most of which are not tested as part of any single study. These factors can include statistical power, sample size, variations in experimental protocol, or situational factors unique to the particular context of the study. Identifying such variability is what multi-lab collaborations can contribute - they have all the advantages of preregistered studies, but add additional information about the magnitude and consistency of the result.

Multi-lab collaborations assessing the veracity of promising educational interventions prior to widespread implementation could provide real value to education. Multi-lab collaborations may sound similar to traditional applications of meta-analysis, a tool to aggregate 
a body of work on a topic - sometimes hundreds of studies. Multi-lab collaborations often make use of meta-analytic methods, but because traditional meta-analyses include studies conducted across time, often by researchers applying different interventions with differing applications or protocols, and strongly affected by publication bias, the ability of meta-analysis to identify the true effect is inherently limited. Among other limitations (e.g., Lakens, Hilgard, \& Staaks, 2016), traditional meta-analyses can rarely answer the question of how much the variability in findings - or variability in replicability - can be attributed to sample, researcher, statistical power, or bias. Multi-lab collaborations can. Moreover, multi-lab collaboration results are almost always subjected to meta-analysis as a final step. However, the meta-analysis results from a multi-lab collaborations are inherently more trustworthy because (a) they are (typically) preregistered, (b) all results are included (no grey literature), and (c) they rely on primary studies that used highly similar protocols, eliminating irrelevant sources of between-study variance.

Research on the instructional strategy of Reciprocal Teaching is a good example of how challenging it can be to produce credible meta-analytic estimates of results produced outside the context of a multi-lab collaboration. In Reciprocal Teaching, a cognitive strategy is modelled with the goal of students applying the strategy to novel content. Reciprocal Teaching strategies often take the form of summarizing, questioning, and predicting in analyzing text. Two metaanalyses found mean effects of Reciprocal Teaching to be $d=.32$ to $d=.88$ (Galloway, 2003; Rosenshine \& Meister, 1994). However, sometimes the intervention was delivered in small groups as opposed to whole classes. In some studies, the outcome measure was a state standardized test, whereas in others it was a teacher-made test. These are all variations in protocol or intervention application whose unique effects cannot be tested via meta-analysis unless multiple studies implemented the same protocol. Multi-lab collaborations controls for 
extraneous factors to systematically estimate the true effect and the conditions under which that effect manifests. By having multiple research sites and collaborators, a single multi-lab "study" can reveal what factors influence the effect better than a meta-analysis of haphazardly conducted existing studies. These findings are more likely to capture the true effect of this instructional method compared to any single study conducted by one researcher. Although multi-lab collaboration requires a lot of coordination, if multiple independent teams find consistent effects in both direction and magnitude, that serves as a stronger foundation for educational implementation than a single small-scale study. Moreover, multi-lab collaborations achieve such cross-lab generalizability estimates more quickly than serial independent teams working on projects.

\section{Persistent Collaboration}

As mentioned above, the creation of multi-lab collaborations may be difficult, particularly for researchers who are not well-established within the field and/or those at institutions with fewer research resources. However, there are new initiatives within psychology that may be useful to leverage in education research. PsyAccelerator, (https://psysciacc.org) is a network of psychology research labs (over 400 as of March 2019) from 50 countries from every populated continent. According to its website, its mission is "to accelerate the accumulation of reliable and generalizable evidence in psychological science, reducing the distance between truth about human behavior and mental processes and our current understanding." Its founder views PsyAccelerator as psychology's equivalent of CERN, home of the Large Hadron Collider mentioned above (Chartier, 2017). In essence, it is an infrastructure to create multi-lab collaborations to facilitate persistent large-scale collaboration. 
Similarly, StudySwap (https://osf.io/view/StudySwap/) is a platform on which researchers can offer and request assistance in data collection (either whole studies or additional participants; Chartier, Riegleman, \& McCarthy, 2018). Using StudySwap, researchers can post "Haves" or "Needs" as part of an online exchange. Both StudySwap and PsyAccelerator help crowdsource and coordinate resources so that an individual researcher is not responsible for all aspects of the research cycle (or collecting all data). Broadly, persistent collaboration consortia could help more efficiently allocate existing resources, reduce barriers to entry, and increase inclusivity, while also increasing transparency, rigor, and reliability of the results produced (Uhlmann, et al., 2018). Such an infrastructure may also facilitate answering questions that may otherwise not be possible (e.g., by increasing statistical power, increasing diversity of the sample, ability to assess sub-groups that are often too small). As discussed below in the section on Preregistered Adversarial Collaborations, persistent collaboration consortia would shift when arguments over what is appropriate or persuasive take place. Such debate already occurs in research, but typically after data have been collected and it is often too late to fix the issues without allocating more resources to collect additional data.

Education could (and we argue, should) create initiatives similar to StudySwap and PsyAccelerator. A StudySwap for education research could facilitate data collection from hard to reach populations that may not be concentrated within schools or districts (e.g., valedictorians, students who've been suspended, students with low-incidence disabilities such as visual impairments). For an EduAccelerator, imagine 50 research teams across the country forming a research consortium. Any researcher, regardless of career status or university affiliation is also able to join. Each year, consortia establish their priorities, determine what data to collect, and how to analyze it. Creating the infrastructure takes resources, but the pay off over time would be 
at both the micro (individual consortia member) and macro (across the consortia) levels. Each contributing school learns something about itself while also contributing to a larger project that informs them (and the world) whether the results from any individual consortia member are the exception or the norm. Knowing how their specific context fits within the larger spectrum of results could have immense value for schools. It could help them select and prioritize specific interventions as well as help them set realistic expectations for effects of those interventions in their specific environment. In non-crowdsourced research, constraints on generality of results (Simons, Shoda, \& Lindsay, 2017) can be guesswork. In crowdsourced research, diverse consortia provide clear estimates of constraints on generality as part of the typical process. As stated above, StudySwap and PsyAccelerator exist within psychology; no such persistent collaborative consortia exists yet within education. That said, the primary barrier to creating such an infrastructure is user interest and engagement, both of which are within the field's control.

\section{Collaborative Analysis}

The benefits of collaboration can also be leveraged specifically within the data analysis stage of the research process. In this approach multiple research teams independently analyze the same dataset to answer the same research question. Analogous to a specification curve (Simonsohn, Simmons, \& Nelson, 2015) or multiverse analysis (Steegen et al., 2016) conducted by an individual researcher, this model helps reduce the impact of analytic flexibility by making it transparent.

A key revelation from recent meta-science research is the extent to which data analytic choices influence subsequent research results. This can happen even in the absence of $p$-hacking or other QRPs (Silberzahn et al., 2018). Every decision made concerning missing data, outlier exclusions, assumption testing, variable aggregation and transformation, model selection, 
covariates, etc., comes with a host of alternative decisions could have been made, any number of which might have been equally reasonable. One way to assess (and address) the magnitude of this variability is by having multiple analysts interrogate the same dataset while testing the same hypothesis - all with an eye toward understanding how differing methodological choices influence the results.

For example, Silberzahn et al. (2018) reported findings from a project in which 29 independent research teams analyzed the same data to determine whether soccer referees gave more red cards to dark-skinned players. Teams differed substantially in the choices they made, resulting in 20 statistically significant and 9 null findings, with effect sizes ranging from moderately large to practically nil and varying confidence interval width estimates. Schweinsberg et al. (2018) extended this model to crowdsource not only the analysis of a particular dataset, but also the hypotheses tested with it. Additionally, the 49 participating research teams were given freedom to decide how to operationalize key variables within the dataset (whereas the Silberzahn project leaders settled a priori on number of red cards as a specific operationalization of discrimination). Effects varied in both magnitude and direction, suggesting the impact of analytic flexibility is a function of both the data's complexity and the specificity of the hypothesis and measurement.

Crowdsourced analysis of a dataset can also be combined with the Registered Report format (where a study's literature review, method, and analytic plan are reviewed prior to data collection; https://cos.io/rr/). Hussey et al. (2018) announced the release of a massive dataset that includes over 444,000 observations from 200,000 participants on 15 commonly used individual difference measures. The authors made $15 \%$ of the dataset publicly available for exploratory analysis, along with the data codebooks and collection procedures (https://osf.io/pcjwf/). 
Research teams can use the exploratory data to help form hypotheses and data analysis plans that can then be submitted to a journal as a Registered Report. If granted in-principle acceptance, researchers will be given access to the rest of the data to conduct confirmatory analyses. As of February 2019, fourteen journals have agreed to accept Registered Reports based on this data. This model could easily be applied to data routinely collected within schools for state, national, and international assessments. For example, a journal could put out a call for Registered Reports that would all rely on the next release of PISA or NAEP data, and/or researchers could agree on a set of hypotheses to test with these datasets a priori. The model further extends to qualitative research, as multiple analysts can facilitate triangulation of thematic or theoretical codes drawn from interview transcripts, open-ended survey responses, classroom observations, videos, social media posts, or student work products.

In short, processing and modeling options abound for education data, and using multiple analysts helps ensure results are robust across those decisions. This broadens the scope of what can be learned by any set of data, and perhaps more importantly, clarifies what cannot be known by revealing the uncertainty of findings resulting from analytic flexibility.

\section{Preregistered Adversarial Collaborations}

The previously discussed collaborative models are built on researchers agreeing on many fundamental premises, such as what worked and what is worth testing going forward. Clearly this is not always the case. A fifth collaborative research model is built on pre-existing disagreement within the field. A preregistered adversarial collaboration (PAC) is a joint research project conducted by individuals or research teams who disagree about an important theoretical or empirical question. This kind of collaboration was suggested by Latham, Erez, and Locke in 1988 and was more recently advocated by Kahneman (2003) as an alternative to the protracted 
stalemate of the critique-reply-rejoinder method seen in academic journals. PACs are particularly useful in the field of education, where long-running disputes stretch across years, if not decades (e.g., whether students should be grouped by age or readiness).

Several researchers, mostly from the field of psychology, have clarified the components of a successful PAC (Kerr, Ao, Hogg, \& Zhang, 2018; Matzke et al, 2015; Mellers, Hertwig, \& Kahneman, 2001; Neir \& Campbell, 2013). Ideally collaborators should have a sincere desire to address differences, be objective, and be open to change. Even with willing collaborators it is advisable to involve another individual who can arbitrate any differences that arise. This arbitrator should be sufficiently knowledgeable about the theories and methods involved and be someone who both sets of collaborators trust to be impartial.

PACs begin with a discussion of the major theoretical differences and possible areas of disagreement to generate testable hypotheses (Latham, Erez, \& Locke, 1988). It is possible that the areas of disagreement have been unintentionally overstated and the researchers should first tease apart rival explanations. The initial discussion is a success if it generates testable hypotheses about important issues.

Next, collaborators jointly create a plan of study including design, data collection, analysis, and reporting of findings. Exhaustive planning is important to ensure true social collaboration and because of the large number of researcher degrees of freedom that occur during scientific enquiry (Simmons, et al., 2011; Wicherts et al, 2016). Collaborators should anticipate possible interpretations of the outcomes and explicitly identify what kind of results would be consistent/inconsistent with their expectations. Namely, what results would change or falsify beliefs. If no result would falsify beliefs, it is not a scientific disagreement and empirical 
assessment would not be fruitful. Finally, the entire research protocol is preregistered prior to data collection.

Preregistered adversarial collaboration makes the research process transparent and has the potential to resolve disagreements that could otherwise take years, allowing the field to move forward to new disputes. PACs not only prevent possible QRPs (e.g., p-hacking in order to achieve a desired result), but also highlight positive examples of excellent programming and research (Fiedler, 2018). Rather than disagreeing parties serially waiting to point out the flaws of the other sides research, the two groups agree on what would (or could) be informative a priori, thus touting the potential of resolving a disagreement that could otherwise drag on for years. PACs also help prevent researchers from overselling results as easily because the nature of the disagreement helps prevent groupthink.

PACs have great potential, but expectations should be reasonable. The process begins with a substantial disagreement and it is possible that there will not be a complete resolution. Discussion of these disagreements are often part of the co-authored paper. Although the preregistered protocol should eliminate post hoc interpretation of effects (how various patterns of results will be interpreted should be agreed upon in advance), thinking about post hoc explanations is unavoidable. Researchers can agree to jointly plan follow-up research based on the results of the initial collaboration. The process narrows differences and increases mutual respect. In fact, PAC was recently used to address the differing explanations posited by the original researchers and the replicators for a failed replication (Coles, March, MarmolegoRamos, Banaruee, Butcher, et al, 2019). Bringing diverse perspectives to the table at the research design stage ensures that concerns from multiple sides are incorporated into the research process. 
There are numerous educational issues and programs that would benefit from research that incorporates the concerns of both proponents and skeptics. For example, the implementation of restorative justice is gaining popularity in school systems worldwide, but research is far behind practice (Song \& Swearer, 2016). Restorative justice as applied to schools is a philosophy of discipline that focuses on rebuilding relationships in the school community by bringing together offenders and those affected by the infraction to decide together how to repair the harm caused to others (Suvall, 2009; Zehr, 2015). Although some scholars and policy makers support the potential of restorative justice (Pavelka, 2013; Wearmouth, Mckinney, \& Glynn, 2007; Zehr, 2015), reports from school districts suggest that restorative justice can negatively affect school climate, safety, and staff morale (Augustine, Engberg, Grimm, Lee, Wang, et al, 2018; Eden, 2019; Gray, Sirinides, Fink, Flack, DuBois, et al, 2017). Main areas of disagreement regarding restorative justice include the relative importance of prescriptive policies, requirement of adherence to philosophical values, the level of necessary implementation in a school, and the role that restorative justice plays in addressing issues of racial equality (Anfara, Evans, \& Lester, 2013; Morrison \& Vaandering, 2012; Song \& Swearer, 2016). Rather than debate for decades while millions of students age through the system, researchers supporting the potential of restorative justice can collaborate with those who are skeptical to subject their perspectives to an empirical test through the production of joint research under an agreed upon protocol. The researchers would define ahead of time what type of results adequately address their differences in perspective. More philosophically, we believe greater numbers of stakeholder groups who have different roles or viewpoints engaging with each other would generate numerous benefits, even if little collaborative research is produced. 
When educational initiatives do not produce the expected results, researchers may explain this away as a lack of fidelity to the process in the field (Kaimal \& Jordan, 2016; Missett \& Foster, 2015). Repeatedly producing research that does not answer essential questions is a waste of researcher time and resources. For example, it has been difficult to determine the effectiveness of integration of instructional technology in the classroom because of variance in commitment to learning new teaching methods (Delgado, Wardlow, McKnight, \& O’Malley, 2015; Nicol, Owens, Le Coze, MacIntyre, \& Eastwood, 2018). Researchers can collaborate ahead of time to define sufficient level of fidelity and how fidelity can best be measured. This can greatly diminish the post-hoc critique and goalpost-moving that researchers often use to maintain favored beliefs in the presence of disconfirmatory evidence. Adversarial collaboration can bring together educational theorists, applied researchers, and policy makers to create a research protocol that produces results that are considered persuasive by all stakeholders.

\section{Discussion}

In this paper, we proposed that education research, as typically practiced, does not always live up to its scientific aspirations and would benefit from greater adoption of collaborative research methods. Each of the collaborative models discussed would also reduce researcher degrees of freedom and the ability to use QRPs and most would help differentiate general effects from context-specific effects. Collaborative initiatives would improve what we know about educational contexts as well as what results are generalizable, when, and how while shrinking the scale of resources uses and time needed to obtain this information. Additionally, many of these initiatives will help assist under-resourced programs and investigators who would otherwise be unable to conduct independent large-scale evaluation. It is our belief that when the field adopts 
these types collaborative models, the field will more credibly answer research questions and earn the trust of practitioners and policymakers.

To be fair, some positive strides have already been made in collaborative educational research. For example, the "ManyClasses" project is an application of the multi-lab collaboration concept across K-12 classrooms. Their stated goal is to "examine the same research question in dozens of contexts, spanning a range of courses, institutions, formats, and student populations" (manyclasses.org). This is the kind of collaborative, highly-informational research that is needed. Similarly, after finding a "developer effect" where studies included in the What Works Clearinghouse that had been commissioned by the intervention developer averaged 1.5 times the effect size of independently conducted evaluations, Wolf and colleagues (2019) suggested that requiring preregistration should become necessary for inclusion in the What Works Clearinghouse.

The trope of comparing modern classrooms to those from 100 years ago is almost a cliché. But what about comparing modern education research practices with those of 100 years ago? How much have educational researchers taken advantage of advances in research methods in what they do? As illustrated above, many other scientific fields have had great success implementing the proposed collaborative research models. We believe much of this success has potential to transfer to education research as well.

We are not proposing that all undergraduate honors theses should become large scale collaborations (although what a great learning opportunity for undergraduate students! $!^{\mathrm{i}}$ ). Similarly, the role and importance of small scale studies developing and testing new ideas remains extremely high. That said, we believe that the more important the issue, the more resources we should put behind to understanding what it is, what causes it, and what can expand, 
reduce, or remove it. Obviously, "importance" is not universally agreed upon. What may be important to some may be less important to others. Regardless, similar to the current research model, individual researchers, schools, and funders would all still be able to rely on their own values systems to determine which collaborative research projects they choose to participate in. Factors such as personal interest/relevance, expected magnitude of effect, the number of students affected, and many others would all continue to play important roles when selecting research project priority. The primary change would be in the scale collaborative research projects undertake, leading to an expected synergistic return in the value derived from the subsequent results.

The proposed practices will not solve all problems, but that does not mean that we should not use them. To be successful, such initiatives will need to be relevant. Moreover, because they are new, bumps in the road should be expected as applicability to education research is assessed. Which projects are best suited for these types of projects is up for debate; it may be best to start with relatively low-hanging opportunities so that all involved can learn the process before advancing to more complicated topics.

As discussed elsewhere (Uhlmann et al., 2018), we envision collaborative research serving as a complementary addition to traditional independent research. Substantial exploratory, descriptive, and confirmatory work could still be primarily independently conducted, although all could also be the focus of collaborative research. For example, large-scale collaborative qualitative projects could assess the generalizability of some qualitative work (e.g., do independent researchers analyzing the same data develop similar themes? If not, what does that say about the value of the findings?). 
Importantly, adoption of large scale collaborative research in education may also require a shift in incentive alignment for individual researchers as well as many other stakeholders. Becoming famous should not be our goal as researchers, but is often associated with many professional perks. Without buy-in from hiring and promotion committees and funders, shifting to a more large-scale collaborative research model will be quite difficult. The existing research model in education has emphasized number of studies. Most researchers need to publish order to be promoted and this model prioritizes quantity over quality. Many of the collaborative methods discussed in this paper flip that model on its head. All of these collaborations would take additional coordination, but they would also yield far more information than the current model of academic research.

\section{Conclusion}

We often seek to have students transfer what they learn in the school environment to other life situations while also learning from observing the actions of others and working well with others. As researchers, we should hold ourselves to (at least) the same standard. Other fields are developing new ways to improve research quality; education can benefit from their efforts and improve the quality of research produced. Greater implementation of large-scale collaboration has the potential to provide valuable information about direction, magnitude, and generalizability of effects in an efficient manner. With this improved quality, we may start to earn the trust of practitioners and policymakers.

\footnotetext{
${ }^{\mathrm{i}}$ For an example of a large scale collaborative research project involving undergraduate research experience, see: For an example, see:

https://osf.io/wfc6u/wiki/home/?_ga=1.235318916.446840397.1471873348.
} 


\section{References}

Aad, G., Abajyan, T., Abbott, B., Abdallah, J., Abdel Khalek, S., Abdelalim, A. A., ... Abolins, M. (2012). Observation of a new particle in the search for the Standard Model Higgs boson with the ATLAS detector at the LHC. Physics Letters B, 716, 1-29. doi:10.1016/j.physletb.2012.08.020

Abbott, B. P., Abbott, R., Abbott, T. D., Acernese, F., Ackley, K., Adams, C., ... \& Affeldt, C. (2017). GW170817: Observation of gravitational waves from a binary neutron star inspiral. Physical Review Letters, 119, 161101.

Anderson, S. F., \& Maxwell, S. E. (2017). Addressing the "Replication Crisis": Using original studies to design replication studies with appropriate statistical power. Multivariate Behavioral Research, 52, 305-324. doi:10.1080/00273171.2017.1289361

Anderson, D., Spybrook, J., Maynard, R. (2019). REES: A registry of efficacy and effectiveness studies in education. Educational Researcher, 48, 45-50. doi: https://doi.org/10.3102/0013189X18810513

Anfara, V. A., Evans, K. R., \& Lester, J. N. (2013) Restorative justice in education: What we know so far. Middle School Journal, 44(5), 57-63, doi:10.1080/00940771.2013.11461873

Aschwanden, C. (2015). Science isn't broken: It's just a hell of a lot harder than we give it credit for. Retrieved from: https://fivethirtyeight.com/features/science-isnt-broken/

Augustine, C. H., Engberg, J., Grimm, G. E., Lee, E., Wang, E. L., Christianson, K, \& Joseph, A. A. (2018). Can restorative practices improve school climate and curb suspensions? An evaluation of the impact of restorative practices in a mid-sized urban school district. 
Santa Monica, CA: RAND Corporation.

https://www.rand.org/pubs/research_reports/RR2840.html.

Baker M. (2016). Is there a reproducibility crisis? Nature 533, 453-454.

Camerer, C. F., Dreber, A., Holzmeister, F., Ho, T.-H., Huber, J., Johannesson, M., ... Wu, H. (2018). Evaluating the replicability of social science experiments in Nature and Science between 2010 and 2015. Nature Human Behaviour, 2, 637-644. doi:10.1038/s41562018-0399-z

Chase, L. J., \& Chase, R. B. (1976). A statistical power analysis of applied psychological research. Journal of Applied Psychology, 61, 234-237. doi:10.1037/0021-9010.61.2.234

Chartier, C. (2017). Building a distributed CERN for psychological science. Retrieved from: https://christopherchartier.com/2017/08/26/building-a-cern-for-psychological-science/

Chartier, C. R. , Riegleman, A., \& McCarthy, R. J. (2018). StudySwap: A platform for interlab replication, collaboration, and resource exchange. Advances in Methods and Practices in Psychological Science, 1, 574-579. https://doi.org/10.1177/2515245918808767

Chatrchyan, S., Khachatryan, V., Sirunyan, A. M., Tumasyan, A., Adam, W., Aguilo, E., ... Fabjan, C. (2012). Observation of a new boson at a mass of $125 \mathrm{GeV}$ with the CMS experiment at the LHC. Physics Letters B, 716, 30-61.

doi:10.1016/j.physletb.2012.08.021

Cohen, J. (1988). Statistical Power Analysis for the Behavioral Sciences (2nd ed.). Hillsdale, NJ Lawrence Erlbaum Associates, Publishers.

Coles, N. A., March, D. S., Marmolejo-Ramos, F., Banaruee, H., Butcher, N., Cavallet, M., ... Marozzi, M. (2019, February 4). The Many Smiles Collaboration: A Multi-Lab 
Foundational Test of the Facial Feedback Hypothesis.

https://doi.org/10.31234/osf.io/cvpuw

Cook, B. G., Lloyd, J. W., Mellor, D., Nosek, B. A., \& Therrien, W. J. (2018). Promoting open science to increase the trustworthiness of evidence in special education. Exceptional Children, 85, 104-118. doi: 10.1177/0014402918793138

Crede, M., Tynan, M. C., \& Harms, P. D. (2017). Much ado about grit: A meta-analytic synthesis of the grit literature. Journal of Personality and Social Psychology, 113, 492511. doi: http://dx.doi.org/10.1037/pspp0000102

de Vries Y. A., Roest, A.M., de Jonge, P., Cuijpers, P., Munafò, M. R., \& Bastiaansen, J.A. (2018). The cumulative effect of reporting and citation biases on the apparent efficacy of treatments: the case of depression. Psychological Medicine, 1-3. https://doi.org/ $10.1017 / \mathrm{S} 0033291718001873$

Delgado, A. J., Wardlow, L., McKnight, K., \& O’Malley, K. (2015). Educational technology: A review of the integration, resources, and effectiveness of technology in K-12 classrooms. Journal of Information Technology Education: Research, 14, 397-416. http://www.jite.org/documents/Vol14/JITEv14ResearchP397-416Delgado1829.pdf

Eden, M. (2019, January 14). Restorative justice isn't working, but that's not what the media is reporting. Retrieved from: https://edexcellence.net/articles/restorative -justice-isntworking-but-thats-not-what-the-media-is-reporting

Fiedler, K. (2018). The creative cycle and the growth of psychological science. Perspectives on Psychological Science, 13, 433 -438. https://doi.org/10.1177/1745691617745651

Fiedler, K., \& Schwarz, N. 2015. Questionable research practices revisited. Social Psychological \& Personality Science, 7, 45-52. 
Flake, J. K., \& Fried, E. I. (2019). Measurement schmeasurement: Questionable measurement practices and how to avoid them. https://doi.org/10.31234/osf.io/hs 7wm

Francis, G., Tanzman, J., \& Matthews, W. J. (2014) Excess success for psychology articles in the journal science. PLOS ONE 9(12): e114255. doi: 10.1371/journal.pone.0114255

Fraser, H., Parker, T., Nakagawa, S., Barnett, A., \& Fidler, F. (2018). Questionable research practices in ecology and evolution. PLoS ONE 13(7): e0200303.

Galloway, A. M. (1994). Improving reading comprehension through metacognitive strategy instruction: Evaluating the evidence of effectiveness of the reciprocal teaching procedure (Unpublished doctoral dissertation). University of Nebraska, Lincoln, NE.

Gelman, A., \& Carlin, J. (2014). Beyond power calculations: Assessing Type S (sign) and Type M (magnitude) errors. Perspectives on Psychological Science, 9, 641-651. DOI: $10.1177 / 1745691614551642$

Gelman, A., \& Loken, E. (2013). The garden of forking paths: Why multiple comparisons can be a problem, even when there is no "fishing expedition" or "p-hacking" and the research hypothesis was posited ahead of time. Unpublished manuscript.

Gray, M., Sirinides, P. M, Fink, R, Flack, A., DuBois, T. Morrison, K., \& Hill, K. (2017). Discipline in Context: Suspension, Climate, and PBIS in the School District of Philadelphia. CPRE Research Reports. Retrieved from http://repository.upenn.edu/cpre_researchreports/106

Gehlbach, H. \& Robinson, C. D. (2018). Mitigating illusory results through preregistration in education. Journal of Research on Educational Effectiveness. 11, 296-315. DOI: $10.1080 / 19345747.2017 .1387950$ 
Greenberg, E. (2018). New measures of student poverty: Replacing free and reduced-price lunch status based on household forms with direct certification. Retrieved from: https://www.urban.org/research/publication/new-measures-student-poverty

Hahn, G. J., \& Meeker, W. Q. (1993). Assumptions for statistical inference. The American Statistician, 47, 1-11. doi:10.1080/00031305.1993.10475924

Harwell, M. \& LeBeau, B. (2010). Student eligibility for a free lunch as an SES measure in education research. Educational Researcher, 39, 120-131. doi:10.3102/0013189X10362578

Hussey, I., Hughes, S., Lai, C. K., Ebersole, C. R., Axt, J. R., \& Nosek, B. A. (2018). Attitudes 2.0: A large dataset for investigating relations among implicit and explicit attitudes and identity. Unpublished Manuscript. Retrieved from https://osf.io/pcjwf

International Human Genome Sequencing Consortium. (2001). Initial sequencing and analysis of the human genome. Nature, 409(6822), 860-921. doi:10.1038/35057062

Ioannidis, J. P. A. (2005). Why most published research findings are false. PLoS Medicine, 2, 696-701. doi:10.1371/journal.pmed.0020124

John, L. K., Loewenstein, G., \& Prelec, D. (2012). Measuring the prevalence of questionable research practices with incentives for truth telling. Psychological Science, 23, 524-532. doi:10.1177/0956797611430953

Kahneman, D. (2003). Experiences in collaborative research. American Psychologist, 58, 723 730. http://dx.doi.org/10.1037/0003-066X.58.9.723

Kaimal, G. \& Jordan, W. J. (2016). Do incentive-based programs improve teacher quality and student achievement? An analysis of implementation in 12 urban charter schools. Teachers College Record, 118, 1-34. 
Kerr, N. L., Ao, X., Hogg, M. A., \& Zhang, J. (2018). Addressing replicability concerns via adversarial collaboration: Discovering hidden moderators of the minimal intergroup discrimination effect. Journal of Experimental Social Psychology, 78, 66 -76. http://dx.doi.org/10.1016/j.jesp.2018.05.001

Klein, Ratliff, Vianello, Adams Jr., Bahnik, Bernstein, et al. (2014). Investigating variability in replicability: A “many labs" replication project. Social Psychology, 45, 142-152. doi:10.1027/1864-9335/a000178

Lakens, D., Hilgard, J., \& Staaks, J. (2016). On the reproducibility of meta-analyses: Six practical recommendations. BMC Psychology, 4. https://doi.org/10.1186/s40359-0160126-3

Latham, G. P. Erez, M., \& Locke, E. A. (1988). Resolving scientific disputes by the joint design of crucial experiments by the antagonists: Application to the Erez-Latham dispute regarding participation in goal setting. Journal of Applied Psychology, 73, $753-772$. http://dx.doi.org/10.1037/0021-9010.73.4.753

Lindsay, D. S. (2015). Replication in psychological science. Psychological Science, 26, $1827-$ 1832. doi:10.1177/0956797615616374

Lortie-Forgues, H. \& Ingles, M., (2019). Most rigorous large-scale educational RCTs are uninformative: Should we be concerned? Educational Researcher. doi:10.3102/0013189X19832850

Makel, M. C. \& Plucker, J. A. (2014). Facts are more important than novelty: Replication in the education sciences. Educational Researcher, 43, 304-316.

doi:10.3102/0013189X14545513 
Matzke, D., Nieuwenhuis, S., van Rijn, H., Slagter, H. A., van der Molen, M. W., \& Wagenmakers, E-J. (2015). The effects of horizontal eye movement on free recall: A preregistered adversarial collaboration. Journal of Experimental Psychology-General, 144, e1 - e15. https://doi.org/10.1037/xge0000038

McBee, M. T., Makel, M. C., Peters, S. J., \& Matthew, M. S. (2018). A call for open science in giftedness research. Gifted Child Quarterly, 62, 374-388. doi:10.1177/0016986218784178

Mellers, B., Hertwig, R., \& Kahneman, D. (2001). Do frequency representations eliminate conjunction effects? An exercise in adversarial collaboration. Psychological Science, 12, 269 - 275. http://dx.doi.org/10.1111/1467-9280.00350

Miller, P. (Director). (1988). Saturday Night Live [Television series]. New York: National Broadcasting Company (NBC).

Missett, T. C. \& Foster, L. H. (2015). Searching for evidence-based practice: A survey of empirical studies on curricular interventions measuring and reporting fidelity of implementation published during 2004-2013. Journal of Advanced Academics, 26, 96 111. https://doi.org/10.1177/1932202X15577206

Morrison, B., \& Vaandering, D. (2012). Restorative justice: Pedagogy, praxis, and discipline. Journal of School Violence, 11(2), 138-155.

Moshontz, H., Campbell, L., Ebersole, C. R., IJzerman, H., Urry, H. L., Forscher, P. S., ... Chartier, C. R. (2018). The psychological science accelerator: Advancing psychology through a distributed collaborative network. Advances in Methods and Practices in Psychological Science, 1, 501-515. doi:10.1177/2515245918797607 
National Academies of Sciences, Engineering, and Medicine 2017. Communicating science effectively: A research agenda. Washington, DC: The National Academies Press. https://doi.org/10.17226/23674.

National Research Council 2002. Scientific Research in Education. Washington, DC: The National Academies Press. https://doi.org/10.17226/10236.

National Science Foundation and Institute for Educational Sciences. (2018). Companion guidelines on replication \& reproducibility in education research. Retrieved from: https://ies.ed.gov/pdf/CompanionGuidelinesReplicationReproducibility.pdf

Neir, J. A \& Campbell, S. D. (2013). Two outsiders' view on feminism and evolutionary psychology: An opportune time for adversarial collaboration. Sex Roles, 69, 503-506. https://doi.org/10.1007/s11199-012-0154-2

Nicol, A. A. M., Owens, S. M., Le Coze, S. S. C. L., MacIntyre, A., \& Eastwood, C. (2018). Comparison of high-technology active learning and low-technology active learning classrooms. Active Learning in Higher Education, 19, 253 - 265. https://doi.org/10.1177/1469787417731176

Open Science Collaboration. (2015). Estimating the reproducibility of psychological science. Science, 349(6251), aac4716-aac4716. doi:10.1126/science.aac4716

Pashler, H., \& Harris, C. R. (2012). Is the replicability crisis overblown? Three arguments examined. Perspectives on Psychological Science, 7, 531-536. doi:10.1177/1745691612463401

Pashler, H., McDaniel, M., Rohrer, D., \& Bjork, R. (2009). Learning styles: Concepts and evidence. Psychological Science in the Public Interest, 9, 105-119. https://doi.org/10.1111/j.1539-6053.2009.01038.x 
Pavelka, S. (2013). Practices and policies for implementing restorative justice within schools. The Prevention Researcher, 20(1), 15-19.

Pigott, T. D., Valentine, J. C., Polanin, J. R., Williams, R. T., \& Canada, D. D. (2013). Outcomereporting bias in education research. Educational Researcher, 42, 424-432. http://doi.org/10.3102/0013189X13507104

Rosenshine, B., \& Meister, C. (1994). Reciprocal teaching: A review of the research. Review of Educational Research, 4, 479-530. doi:10.3102/00346543064004479

Sagan, C. (1997). The demon-haunted world: Science as a candle in the dark. New York, NY: Ballantine Books.

Schimmack, U. (2012). The ironic effect of significant results on the credibility of multiple-study articles. Psychological Methods, 17, 551-566. doi:10.1037/a0029487

Schochet, P. Z. (2008). Statistical power for random assignment evaluations of education programs. Journal of Educational and Behavioral Statistics, 33, 62-87. doi:10.3102/1076998607302714

Schweinsberg, M., Feldman, M., Staub, N., Prasad, V., Ravid, A., van den Akker, O., ... \& Uhlmann, E. (2018). Crowdsourcing data analysis: Gender, status, and science. Manuscript in preparation.

Sedlmeier, P., \& Gigerenzer, G. (1989). Do studies of statistical power have an effect on the power of studies? Psychological Bulletin, 105, 309-316. doi:10.1037/00332909.105.2.309

Silberzahn, R., Uhlmann, E. L., Martin, D. P., Anselmi, P., Aust, F., Awtrey, E., ... \& Carlsson, R. (2018). Many analysts, one data set: Making transparent how variations in analytic 
choices affect results. Advances in Methods and Practices in Psychological Science, 1, 337-356. https://doi.org/10.1177\%2F2515245917747646

Simmons, J., Nelson, L. D., \& Simonsohn, U. (2011). False-positive psychology: Undisclosed flexibility in data collection and analysis allows presenting anything as significant. Psychological Science, 22, 1359-1366. https://doi.org/10.1177/0956797611417632

Simons, S. J., Shoda, Y., \& Lindsay, D. S. (2017). Constraints on Generality (COG): A proposed addition to all empirical papers. Perspectives on Psychological Science, 12, 1123-1128. https://doi.org/10.1177/1745691617708630

Simonsohn, U., Simmons, J. P., \& Nelson, L. D. (November 24, 2015). Specification curve: Descriptive and inferential statistics on all reasonable specifications. Available at SSRN: http://dx.doi.org/10.2139/ssrn.2694998

Sisk, V. F., Burgoyne, A. P., Sun, J., Butler, J. L., \& Macnamara, B. N. (2018). To what extent and under which circumstances are growth mindsets important to academic achievement? Two meta-analyses. Psychological Science, 29, 549-571. doi:10.1177/0956797617739704

Song, S. Y., \& Swearer, S. S. (2016). The cart before the horse: The challenge and promise of restorative justice consultation in schools. Journal of Educational and Psychological Consultation, 26, 313-324. https://doi.org/10.1080/10474412.2016.1246972

Steegen, S., Tuerlinckx, F., Gelman, A., \& Vanpaemel, W. (2016). Increasing transparency through a multiverse analysis. Perspectives on Psychological Science, 11, 702-712. https://doi.org/10.1177\%2F1745691616658637 
Sterling, T. D. (1959). Publication decisions and their possible effects on inferences drawn from tests of significance — or vice versa. Journal of the American Statistical Association, 54, 30-34. doi:10.2307/2282137

Suvall, C. (2009). Restorative justice in schools: Learning from Jena High School. Harvard Civil Rights-Civil Liberties Law Review, 44, 547-569.

Uhlmann, E. L., Chartier, C. R., Ebersole, C. R., Errington, T. M., Kidwell, M., Lai, C. K., ... Nosek, B. A. (2018, August 13). Scientific utopia: III. Crowdsourcing science. Retrieved from: https://doi.org/10.31234/osf.io/vg649

van der Zee, T. \& Reich, J. (2018). Open education science. AERA Open, 4, 1-15. DOI: $10.1177 / 2332858418787466$

Van Elk, M., Matzke, D., Gronau, Q. F., Guan, M., Vandekerckhove, J., \& Wagenmakers, E. J. (2015). Meta-analyses are no substitute for registered replications: a skeptical perspective on religious priming. Frontiers in psychology, 6, 1365. 10.3389/fpsyg.2015.01365

Vazire, S. (2018). Implications of the credibility revolution for productivity, creativity, and progress. Perspectives on Psychological Science, 13, 411-417. doi:10.1177/1745691617751884

Waterhouse, L. (2006). Multiple intelligences, the Mozart Effect, and emotional intelligence: A critical review. Educational Psychologist, 31, 201-225. doi:10.1207/ s15326985ep4104_1

Wearmouth, J., Mckinney, R., \& Glynn, T. (2007). Restorative justice in schools: A New Zealand example. Educational Research, 49, 37-49. doi:10.1080/00131880701200740 
Wicherts, J. M., Veldkamp, C. L. S., Augesteijn, H. E. M., Bakker, M., van Aert, R. C. M., \& van Assen, M. A. L. M. (2016). Degrees of freedom in planning, running, analyzing, and reporting psychological studies: A checklist to avoid $p$-hacking. Frontiers in Psychology, 7, 1832. https://doi.org/10.3389/fpsyg.2016.01832

Wolf, R., Morrison, J., Slavin, R., Risman, K. (2019). Do developer-commissioned evaluations inflate effect sizes? Presented at the annual meeting of SREE in Washington DC. Retrieved from: https://hechingerreport.org/wp-content/uploads/2019/03/developerabstract.pdf

Yong, E. (2018). Psychology's replication crisis is running out of excuses. Retrieved from: https://www.theatlantic.com/science/archive/2018/11/psychologys-replication-crisisreal/576223/

Zehr, H. (2015). The little book of restorative justice: Revised and updated. Intercourse, PA: Good Books. 
Table 1

Collaborative research initiatives, their benefits, and example resources

\begin{tabular}{|c|c|c|}
\hline Solution & Benefits & Resources \& Examples \\
\hline $\begin{array}{l}\text { Participating } \\
\text { Labs Run } \\
\text { Different } \\
\text { Studies }\end{array}$ & $\begin{array}{l}\text { Assess credibility of } \\
\text { previous studies }\end{array}$ & $\begin{array}{l}\text { Open Science Collaboration: } \\
\text { https://osf.io/vmrgu/ }\end{array}$ \\
\hline $\begin{array}{l}\text { Multi-lab } \\
\text { Collaborations }\end{array}$ & $\begin{array}{l}\text { Informs about } \\
\text { generalizability, } \\
\text { heterogeneity of effect }\end{array}$ & $\begin{array}{l}\text { Manyclassess.org } \\
\text { https://osf.io/wx7ck/ } \\
\text { Overview of ManyLabs2: https://cos.io/our- } \\
\text { services/research/many-labs-2-project-overview/ }\end{array}$ \\
\hline $\begin{array}{l}\text { Persistent } \\
\text { Collaboration }\end{array}$ & $\begin{array}{l}\text { Facilitate large scale } \\
\text { data accumulation }\end{array}$ & $\begin{array}{l}\text { PsyAccelerator: https://psysciacc.org } \\
\text { StudySwap: https://osf.io/view/StudySwap/ }\end{array}$ \\
\hline $\begin{array}{l}\text { Collaborative } \\
\text { Analysis }\end{array}$ & $\begin{array}{l}\text { Makes analytic } \\
\text { flexibility transparent } \\
\text { and develops consensus } \\
\text { assessment }\end{array}$ & $\begin{array}{l}\text { ManyAnalysts, One Dataset: } \\
\text { https://psyarxiv.com/qkwst/ }\end{array}$ \\
\hline $\begin{array}{l}\text { Preregistered } \\
\text { Adversarial } \\
\text { Collaboration }\end{array}$ & $\begin{array}{l}\text { Reduces post hoc } \\
\text { "Whataboutism" and } \\
\text { provides clarity } \\
\text { regarding a program's } \\
\text { strengths and weakness }\end{array}$ & $\begin{array}{l}\text { Example Adversarial Collaboration Agreement: } \\
\text { https://osf.io/deany } \\
\text { Matzke, D., van Rijn, H., Wagenmakers, E.-J., } \\
\text { Slagter, H., van der Molen, M., \& Nieuwenhuis, } \\
\text { S. (2014, June 3). The effect of horizontal eye } \\
\text { movements on free recall performance. A purely } \\
\text { confirmatory replication study. Retrieved from } \\
\text { osf.io/pxt3m }\end{array}$ \\
\hline
\end{tabular}

